\title{
The Number of Positive Tumor Marker Status Is Beneficial for the Selection of Therapeutic Modalities in Patients with Hepatocellular Carcinoma
}

\author{
Toru Beppu*1,2, Shigeki Nakagawa², Hidetoshi Nitta², Hirohisa Okabe², Takayoshi Kaida², \\ Katsunori Imai ${ }^{2}$, Hiromitsu Hayashi ${ }^{2}$, Yuki Koga ${ }^{1,2}$, Kunitaka Kuramoto ${ }^{1,2}$, \\ Daisuke Hashimoto ${ }^{2}$, Yo-ichi Yamashita², Akira Chikamoto ${ }^{2}$, \\ Takatoshi Ishiko ${ }^{2}$ and Hideo Baba $^{2}$ \\ ${ }^{1}$ Department of Surgery, Yamaga City Medical Center, Kumamoto, Japan; ${ }^{2}$ Department of Gastroenterological Surgery, Graduate \\ School of Life Sciences, Kumamoto University, Kumamoto, Japan
}

\begin{abstract}
Hepatic resection (HR) and radiofrequency ablation (RFA) are popular local therapies for early-stage hepatocellular carcinoma (HCC). Alpha-fetoprotein, Lens culinaris agglutininreactive fraction of alpha-fetoprotein, and des-c-carboxy prothrombin are well-known and useful tumor markers for HCC. The positive number status of these tumor markers has recently been demonstrated as beneficial for predicting outcome for HCC patients treated with local therapy. Although the normal ranges reported have differed by institution, the positivity of tumor markers is consistent and can easily be assessed. Kumamoto and Wakayama's group clearly demonstrated the following: 1) Regardless of the degree of tumor stage, a triple-positive tumor marker profile can predict poor outcome in HCC patients undergoing HR; 2) For RFA alone, HCC patients with double- and triple-positive status, having less than three lesions and lesions $\leq 3 \mathrm{~cm}$ in diameter show comparably insufficient outcomes; 3) For HCC patients with lesions $\leq 5 \mathrm{~cm}$ in Child-Pugh grade $A, H R$ is preferred over RFA; 4) Microvascular invasion rates increased even in the double-positive patients, while poorly differentiated HCC was frequently observed only in the triple-positive patients; and 5) RFA with chemoembolization, anatomical liver resection, and postoperative adjuvant chemoembolization or hepatic arterial chemotherapy might improve the outcome for patients with highly malignant $\mathrm{HCC}$ with multiple positive tumor markers. However, the impacts of these therapies still need to be evaluated in prospective comparative studies.

Citation of this article: Beppu T, Nakagawa $\mathrm{S}$, Nitta $\mathrm{H}$, Okabe $\mathrm{H}$, Kaida T, Imai $\mathrm{K}$, et al. The number of positive tumor marker status is beneficial for the selection of therapeutic
\end{abstract}

Keywords: Triple-positive tumor markers; Double-positive tumor markers; Hepatocellular carcinoma; Hepatic resection; Radiofrequency ablation; Alphafetoprotein; Des- $\gamma$-carboxy prothrombin; Lens culinaris agglutinin reactive fraction of alpha-fetoprotein.

Abbreviations: AFP, alpha-fetoprotein; AFP-L3, lens culinaris agglutinin-reactive fraction of alpha-fetoprotein; DCP, des-c-carboxy prothrombin; DSS, diseasespecific survival; HCC, hepatocellular carcinoma; HR, hepatic resection; HZR, hazard ratio; MI, microvascular invasion; OS, overall survival; RFA, radiofrequency ablation; RFS, recurrence-free survival; TD, rumor differentiation. Received: 26 October 2016; Revised: 11 April 2017; Accepted: 13 April 2017 *Correspondence to: Toru Beppu, Department of Surgery, Yamaga City Medical Center, 511, Yamaga, Kumamoto 861-0593, Japan. Tel: +81-968-44-2185, Fax: +81-968-44-2420, E-mail: tbeppu@kumamoto-u.ac.jp modalities in patients with hepatocellular carcinoma. J Clin Transl Hepatol 2017;5(2):165-168. doi: 10.14218/JCTH. 2016.00055.

\section{Introduction}

Hepatic resection (HR) and radiofrequency ablation (RFA) are common and curative treatments for early-stage hepatocellular carcinoma (HCC). ${ }^{1-3}$ Numerous papers have been published about various tumor markers as indicators of postoperative outcomes. ${ }^{4-10}$ Among them, alpha-fetoprotein (AFP), Lens culinaris agglutinin-reactive fraction of alphafetoprotein (AFP-L3), and des-c-carboxy prothrombin (DCP) are well-known useful tumor markers. Various cutoff levels of these tumor markers have been used; however, the normal limits reported from the various institutes are often different. Thus, optimal cutoff levels cannot be universally applied in studies. On the other hand, the determination of negativity or positivity for tumor markers is quite easy and can be performed regardless of the institutional cutoff values. Recently, the status of three positive tumor markers (AFP, AFP-L3 and DCP) was established as a beneficial prognostic factor for HCC patients treated with HR or RFA. ${ }^{11-15}$

Histological HCC, characterized by features including microvascular invasion (MI) and tumor differentiation (TD), is a great prognostic factor for $\mathrm{HR}^{16-20}$ and RFA. ${ }^{21-24}$ Highly malignant tumors have worse postoperative outcomes; however, the curability of the two treatment modalities might be different according to the grade of tumor malignancy. It has been recently reported that the expression status of positive markers can predict histological tumor malignancy. ${ }^{11,12,14}$

In this review, we have summarized the correlation between the expression number of positive markers and the therapeutic effects of local therapy in HCC patients.

Number of positive tumor markers and prognosis in HCC patients undergoing HR

Two studies have reported on the expression number of positive tumor markers and prognosis in HCC patients treated with $\mathrm{HR}^{11,12}$ (Table 1). The tumor markers studied were AFP, AFP-L3, and DCP. Kiriyama and colleagues ${ }^{11}$ 
Beppu T. et al: Positive tumor marker number status and HCC

Table 1. Positive tumor marker number and outcome in HCC patients who underwent HR and RFA

\begin{tabular}{|c|c|c|c|c|c|c|c|c|c|}
\hline $\begin{array}{l}\text { REF } \\
\text { number }\end{array}$ & Treatment & $\begin{array}{l}\text { Patient } \\
\text { number }\end{array}$ & Classification & $\begin{array}{l}\text { Patient } \\
\text { ratio }\end{array}$ & $\begin{array}{l}\text { 2-year } \\
\text { RFS }\end{array}$ & $\begin{array}{l}\text { 3-year } \\
\text { RFS }\end{array}$ & $\begin{array}{l}\text { 5-year } \\
\text { RFS }\end{array}$ & $\begin{array}{l}\text { 5-year } \\
\text { OS }\end{array}$ & $\begin{array}{l}\text { 5-year } \\
\text { DSS }\end{array}$ \\
\hline \multirow[t]{2}{*}{11} & HR & 199 & $\mathrm{TP}$ & $14 \%$ & & & $17 \%$ & $61 \%$ & \\
\hline & & & Non-TP & $86 \%$ & & & $30 \%$ & $80 \%$ & \\
\hline \multirow[t]{2}{*}{12} & $\mathrm{HR}$ & 185 & $\mathrm{TP}$ & $21 \%$ & $19 \%$ & & & & $36 \%$ \\
\hline & & & Non-TP & $79 \%$ & $38-56 \%$ & & & & $55-83 \%$ \\
\hline \multirow[t]{4}{*}{14} & HR & 136 & TP & $21 \%$ & $60 \%$ & & & $76 \%$ & \\
\hline & & & Non-TP & $79 \%$ & $50-71 \%$ & & & $54-78 \%$ & \\
\hline & RFA & 160 & $\mathrm{TP}$ & $20 \%$ & $27 \%$ & & & $48 \%$ & \\
\hline & & & Non-TP & $80 \%$ & $43-83 \%$ & & & $62-83 \%$ & \\
\hline \multirow[t]{3}{*}{15} & RFA & 160 & $\mathrm{DP}$ & $19 \%$ & & $16 \%$ & & $33 \%$ & \\
\hline & & & TP & $6 \%$ & & $11 \%$ & & $19 \%$ & \\
\hline & & & Non-DP and -TP & $75 \%$ & & $19-30 \%$ & & $78-82 \%$ & \\
\hline
\end{tabular}

Abbreviations: DP, double positive; DSS, disease-specific survival; HCC, hepatocellular carcinoma; HR, hepatic resection; OS, overall survival; REF, reference; RFA, radiofrequency ablation; RFS, recurrence-free survival; TP, triple positive.

evaluated 185 resected HCC patients, known as the Wakayama cohort. Our group also evaluated 199 resected HCC patients that met the Milan criteria, the Kumamoto cohort ${ }^{12}$ which included a larger number of early-stage HCC patients (in comparison with the Wakayama cohort). In the Kumamoto cohort, the number of positive tumor markers was significantly associated with larger tumor size $(>3 \mathrm{~cm})$ and multiple tumors. Based on a qualitative assessment of these three tumor markers, there were $29.6 \%$ and $13.0 \%$ negative, $37.2 \%$ and $40.5 \%$ single positive, $19.1 \%$ and $25.4 \%$ double positive, and $14.1 \%$ and $21.1 \%$ triple positive, respectively in the Kumamoto and Wakayama cohorts.

In the Kumamoto cohort, the 5-year recurrence-free survival (RFS) rate was significantly worse for the triple positive group compared to the non-triple positive group (17.1\% vs. $29.5 \%, p=0.038$ ). The 5 -year overall survival (OS) rates were $61.4 \%$ and $80.2 \%$ for the triple positive and non-triple positive groups, respectively $(p=0.013)$. The 5 -year OS rate was about $20 \%$ worse for the triple positive group. By multivariate analysis, independent predictive factors for poor RFS were positivity for hepatitis $\mathrm{C}$ virus-antibody [hazard ratio $(\mathrm{HZR}), 1.65 ; p=0.015]$, non-initial treatment (HZR, 1.87; $p=0.005)$, and triple positive tumor markers status ( $\mathrm{HZR}$, $1.68 ; p=0.038$ ); and, for poor OS, the independent predictive factors were indocyanine green 15 -min retention rate $>12.6 \%$ $(\mathrm{HZR}, 2.46 ; p=0.0146)$, maximum tumor diameter $>3 \mathrm{~cm}$ (HZR, 2.71; $p=0.004)$, and triple positive tumor markers status (HZR, $2.57 ; p=0.020)$.

In the Wakayama cohort, the 2-year RFS rates were $19.4 \%, 38.2 \%, 55.5 \%$, and $50.7 \%$ in the triple positive, double positive, single positive, and triple negative groups, respectively. The 5 -year disease-specific survival (DSS) rates were $35.9 \%, 54.7 \%, 82.9 \%$, and $62.8 \%$ in the same groups. Multivariate analysis demonstrated that independent risk factors for poor RFS included Child-Pugh class B [HZR, $2.00 ; 95 \%$ confidence interval $(\mathrm{CI}), 1.03-3.87]$, presence of multiple tumors (HZR, 2.53; 95\% CI, 1.73-3.71), and triple positive markers status (HZR, 1.78; 95\% CI, 1.10-2.86); and, for poor DSS, the independent risk factors were presence of multiple tumors ( $\mathrm{HZR}, 2.38 ; 95 \% \mathrm{CI}, 1.31-4.33)$ and triple positive markers status (HZR, 2.41;95\% CI, 1.20-4.83).
Thus, regardless of the degree of tumor stage, a triple positive tumor marker profile was an independent predictive factor for both recurrence and long-term survival in HCC patients who underwent HR.

\section{Number of positive tumor markers and prognosis in HCC patients undergoing RFA}

We recently reported the utility of determining the number of positive tumor markers for HCC patients treated with RFA ${ }^{15}$ (Table 1). A total of 160 patients with less than three lesions and with lesions no more than $3 \mathrm{~cm}$ in diameter were selected and treated with percutaneous, endoscopic or open RFA. The pre-treatment positive rates of these markers were $31.9 \%$, $43.1 \%, 19.4 \%$, and $5.6 \%$ in the negative, single positive, double positive, and triple positive tumor marker groups, respectively. The frequency of triple positive patients was less in the RFA group than in the HR group. ${ }^{11,12}$ Interestingly, in the RFA group, the double and positive tumor marker HCCs provided similarly insufficient outcomes. The 3-year RFS rates were $30 \%, 19 \%, 16 \%$, and $11 \%(p=0.02)$ and the OS rates were $94 \%, 88 \%, 67 \%$, and $37 \%(p<0.001)$ in the negative, single, double, and triple positive groups, respectively. The 2 -year local recurrence rates at the adjacent area of initial ablated lesion were $6.5 \%, 0 \%, 41.2 \%$, and $61.9 \%$, respectively in these groups $(p<0.001)$. By multivariate analysis, a double or triple positive tumor marker status was an independent risk factor for poor OS (HZR, $4.21 ; 95 \% \mathrm{CI}, 1.89-9.37 ; p<0.001)$ and local recurrence (HZR, 5.48; 95\% CI, 2.44-12.33; $p<0.001$ ).

Selection of HR or RFA for HCC patients by assessment of the number of positive tumor markers

The Wakayama group ${ }^{14}$ recently reported the utility of positive conditions of tumor markers for treatment selection of patients with HCC (Table 1). A total of 296 patients with solitary $\mathrm{HCC}$ of $\leq 5 \mathrm{~cm}$ and presenting Child-Pugh grade A (136 HR and 160 RFA) were analyzed. Actually, in the HR group, the frequency of patients undergoing anatomical resection was increased among patients with positive status for three 
tumor markers, while in the RFA group, the frequency of patients treated with RFA following chemoembolization was increased among patients with positive status for three tumor markers.

The 5-year OS rates of HR and RFA were similar, $70.1 \%$ and $69.8 \%$, respectively $(p=0.14)$. Conversely, when we analyzed their outcome according to the positive number of three tumor markers, the 5 -year OS rates were $60.6 \%$, $78.2 \%, 54.2 \%$, and $75.9 \%$ in the HR group, whereas the rates were $83.3 \%, 75.7 \%, 62.2 \%$, and $47.6 \%$ in the RFA group, respectively, in patients with negative, single positive, double positive, and triple positive tumor markers. The $p$-values were different between patients treated with HR and RFA: 0.45 in negative, 0.10 in single positive, 0.77 in double positive, and $<0.01$ in triple positive. By multivariate analysis, RFA application was an independent poor prognostic factor only in the triple positive group (HZR, 1.78; 95\% CI, 1.16-2.72); and, for HCC patients, HR was preferable to RFA for a tumor size of $\leq 5 \mathrm{~cm}$ in Child-Pugh grade $A$.

\section{Number of positive tumor markers and histological malignancy of HCC}

Three studies have reported on the expression number of positive tumor markers and histological HCC malignancy. ${ }^{10,12,14}$ We presented the data from patients with HCC who met the Milan criteria in the Kumamoto cohort ${ }^{12}$ and from patients with solitary $\mathrm{HCC} \leq 5 \mathrm{~cm}$ in diameter in the Wakayama cohort. ${ }^{14}$ Positive data from the Kumamoto cohort is depicted in Fig. $1 .{ }^{12}$ MI rates rose significantly in a stepwise manner according to the increased number of positive tumor markers expressed. For the Kumamoto and Wakayama cohorts, respectively, these were $18.6 \%$ and $16.7 \%$ in the negative group, $27.0 \%$ and $29.3 \%$ in the single positive group, $39.5 \%$ and $46.8 \%$ in the double positive group, and $53.6 \%$ and $56.4 \%$ in the triple positive group.

The percentage of poor TD was significantly increased only in the triple positive group in the Kumamoto cohort (negative, $17.0 \%$; single positive, $17.6 \%$; double positive, $13.2 \%$; triple positive, $46.4 \%$ ). Similarly, tumors representing the Edmondson-Steiner classification grades III or IV were significantly different among the four groups in the Wakayama cohort (negative, $12.5 \%$; single positive, $13.3 \%$; double positive, $25.5 \%$; triple positive, $35.9 \%$ ). The rate of invasive growth showed a stepwise increase among the four groups in the Kumamoto cohort (negative, $0 \%$; single positive, $8.2 \%$; double positive, $10.5 \%$; triple-positive, $17.9 \%$ ).

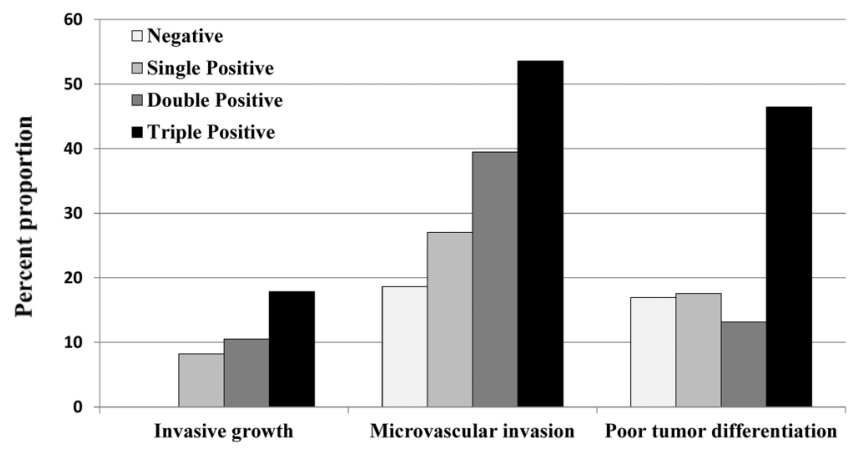

Fig. 1. Association between histological factors and number of positive tumor markers.
Treatment strategy in consideration of the positive tumor marker number profile

The positive tumor marker number profile can clearly estimate recurrence and prognosis in $\mathrm{HCC}$ patients who have undergone local therapy, like HR and RFA. ${ }^{11-15}$

Preoperative assessment for higher recurrence risk allows for planning of an appropriate treatment strategy. According to the histological examination, MI rates increased even in the double positive patients. Both poor differentiation and MI were frequently observed in the triple positive patients. These characteristics were poor prognostic factors after $\mathrm{HR}^{16-20}$ and potent predictors for local recurrence or tumor cell seeding after RFA. ${ }^{21-24}$

The prognosis of HCC after HR was excellent, if the positive number of tumor markers were within a value of 2 . For the RFA-treated group in the Kumamoto cohort, double-positive patients showed equally poor prognosis compared to triplepositive patient. ${ }^{15}$ Conversely, in the Wakayama cohortlimited to double-positive patients-the prognoses were similar in patients undergoing HR and RFA. ${ }^{14}$ For double positive and triple positive patients, if hepatic function is sufficient then HR should be a first-choice therapy, instead of RFA. If hepatic function is insufficient, RFA in combination with chemoembolization might be recommended, which can improve survival over RFA alone. ${ }^{25}$ Prior chemoembolization can reduce arterial blood flow and reduce the heat-sink effect with RFA. ${ }^{26}$ Similarly, RFA plus percutaneous alcohol injection or sorafenib administration can enhance the effect of RFA.

Triple positive tumor marker status was an independent poor prognostic factor even after curative HR. In patients with good liver functional reserve, anatomical HR is advocated based on better outcome for HCC patients with MI. ${ }^{27,28}$ Postoperative adjuvant chemoembolization or hepatic arterial chemotherapy for highly malignant HCC might be useful to reduce recurrence in the remnant liver. ${ }^{29-31}$

In conclusion, preoperative estimation of positive tumor marker number is strongly recommended for HCC patients who undergo local therapy, including HR and RFA.

\section{Conflict of interest}

The authors have no conflict of interests related to this publication.

\section{Author contributions}

Conceived the study and drafted the manuscript (TB, SN, $\mathrm{HN}$ ), performed the analysis and interpretation of data (SN, $\mathrm{HN}, \mathrm{HO}, \mathrm{TK}, \mathrm{KI}, \mathrm{HH}, \mathrm{YK}, \mathrm{KK}, \mathrm{DH}, \mathrm{YY}, \mathrm{AC}$ ), revised the paper for important intellectual content (HB, TI). All authors participated in the acquisition of data and approved the final manuscript.

\section{References}

[1] Cho YK, Kim JK, Kim WT, Chung JW. Hepatic resection versus radiofrequency ablation for very early stage hepatocellular carcinoma: a Markov model analysis. Hepatology 2010;51:1284-1290. doi: 10.1002/hep.23466.

[2] Hasegawa K, Kokudo N, Makuuchi M, Izumi N, Ichida T, Kudo M, et al. Comparison of resection and ablation for hepatocellular carcinoma: a cohort study based on a Japanese nationwide survey. J Hepatol 2013;58:724-729. doi: 10.1016/j.jhep.2012.11.009.

[3] Imai K, Beppu T, Chikamoto A, Doi K, Okabe H, Hayashi H, et al. Comparison between hepatic resection and radiofrequency ablation as first-line 
treatment for solitary small-sized hepatocellular carcinoma of $3 \mathrm{~cm}$ or less. Hepatol Res 2013;43:853-864. doi: 10.1111/hepr.12035.

[4] Toyoda H, Kumada T, Kiriyama S, Sone Y, Tanikawa M, Hisanaga Y, et al. Prognostic significance of simultaneous measurement of three tumor markers in patients with hepatocellular carcinoma. Clin Gastroenterol Hepatol 2006;4:111-117.

[5] Tangkijvanich $\mathrm{P}$, Anukulkarnkusol N, Suwangool P, Lertmaharit S, Hanvivatvong $\mathrm{O}$, Kullavanijaya $\mathrm{P}$, et al. Clinical characteristics and prognosis of hepatocellular carcinoma: analysis based on serum alpha-fetoprotein levels. J Clin Gastroenterol 2000;31:302-308.

[6] Yano $Y$, Yamashita $F$, Kuwaki K, Fukumori $\mathrm{K}$, Kato $\mathrm{O}$, Yamamoto $\mathrm{H}$, et al. Clinical features of hepatitis $\mathrm{C}$ virus-related hepatocellular carcinoma and their association with alpha-fetoprotein and protein induced by vitamin $\mathrm{K}$ absence or antagonist-II. Liver Int 2006;26:789-795. doi: 10.1111/j. 1478-3231.2006.01310.x

[7] Kobayashi M, Ikeda K, Kawamura Y, Yatsuji H, Hosaka T, Sezaki H, et al. High serum des-gamma-carboxy prothrombin level predicts poor prognosis after radiofrequency ablation of hepatocellular carcinoma. Cancer 2009;115: 571-580. doi: 10.1002/cncr.24031.

[8] Kobayashi M, Hosaka T, Ikeda K, Seko Y, Kawamura Y, Sezaki H, et al. Highly sensitive AFP-L3\% assay is useful for predicting recurrence of hepatocellular carcinoma after curative treatment pre- and postoperatively. Hepatol Res 2011;41:1036-1045. doi: 10.1111/j.1872-034X.2011.00858.x.

[9] Saito $Y$, Shimada M, Utsunomiya T, Morine Y, Imura S, Ikemoto T, et al. Prediction of recurrence of hepatocellular carcinoma after curative hepatectomy using preoperative Lens culinaris agglutinin-reactive fraction of alphafetoprotein. Hepatol Res 2012;42:887-894. doi: 10.1111/j.1872-034X. 2012.01004.x.

[10] Masuda T, Beppu T, Horino K, Komori $\mathrm{H}$, Hayashi $\mathrm{H}$, Okabe $\mathrm{H}$, et al. Preoperative tumor marker doubling time is a useful predictor of recurrence and prognosis after hepatic resection of hepatocellular carcinoma. J Surg Oncol 2010;102:490-496. doi: 10.1002/jso.21451.

[11] Kiriyama S, Uchiyama K, Ueno M, Ozawa S, Hayami S, Tani M, et al. Triple positive tumor markers for hepatocellular carcinoma are useful predictors of poor survival. Ann Surg 2011;254:984-991. doi: 10.1097/SLA. ob013e3182215016.

[12] Nakagawa S, Beppu T, Okabe H, Sakamoto K, Kuroki H, Mima K, et al. Triple positive tumor markers predict recurrence and survival in early stage hepatocellular carcinoma. Hepatol Res 2014;44:964-974. doi: 10.1111/hepr. 12277.

[13] Nakagawa S, Hayashi $H$, Nitta H, Okabe $H$, Sakamoto $K$, Higashi T, et al. Scoring system based on tumor markers and Child-Pugh classification for HCC patients who underwent liver resection. Anticancer Res 2015;35: 2157-2163.

[14] Ueno M, Hayami S, Shigekawa Y, Kawai M, Hirono S, Okada K, et al. Prognostic impact of surgery and radiofrequency ablation on single nodular $\mathrm{HCC}$ $\leq 5 \mathrm{~cm}$ : Cohort study based on serum HCC markers. J Hepatol 2015;63: 1352-1359. doi: 10.1016/j.jhep.2015.07.013.

[15] Nitta H, Nakagawa S, Kaida T, Arima K, Higashi T, Taki K, et al. Pre-treatment double- or triple-positive tumor markers are predictive of a poor outcome for patients undergoing radiofrequency ablation for hepatocellular carcinoma. Surg Today 2017;47:375-384. doi: 10.1007/s00595-016-1385-6.

[16] Tsai TJ, Chau GY, Lui WY, Tsay SH, King KL, Loong CC, et al. Clinical significance of microscopic tumor venous invasion in patients with resectable hepatocellular carcinoma. Surgery 2000;127:603-608. doi: $10.1067 / \mathrm{msy}$. 2000.105498
[17] Portolani N, Coniglio A, Ghidoni S, Giovanelli M, Benetti A, Tiberio GA, et al. Early and late recurrence after liver resection for hepatocellular carcinoma: prognostic and therapeutic implications. Ann Surg 2006;243:229-235. doi: 10.1097/01.sla.0000197706.21803.a1.

[18] Oishi K, Itamoto T, Amano H, Fukuda S, Ohdan H, Tashiro H, et al. Clinicopathologic features of poorly differentiated hepatocellular carcinoma. J Surg Oncol 2007;95:311-316. doi: 10.1002/jso.20661.

[19] Imai K, Beppu T, Nakayama $Y$, Ishiko T, Horino K, Komori H, et al. Preoperative prediction of poorly differentiated components in small-sized hepatocellular carcinoma for safe local ablation therapy. J Surg Oncol 2009;100: 121-126. doi: 10.1002/jso.21302.

[20] Choi KK, Kim SH, Choi SB, Lim JH, Choi GH, Choi JS, et al. Portal venous invasion: the single most independent risk factor for immediate postoperative recurrence of hepatocellular carcinoma. J Gastroenterol Hepatol 2011; 26:1646-1651. doi: 10.1111/j.1440-1746.2011.06780.x

[21] Llovet JM, Vilana R, Brú C, Bianchi L, Salmeron JM, Boix L, et al. Increased risk of tumor seeding after percutaneous radiofrequency ablation for single hepatocellular carcinoma. Hepatology 2001;33:1124-1129. doi: 10.1053/ jhep.2001.24233.

[22] Yu HC, Cheng JS, Lai KH, Lin CP, Lo GH, Lin CK, et al. Factors for early tumor recurrence of single small hepatocellular carcinoma after percutaneous radiofrequency ablation therapy. World J Gastroenterol 2005;11: 1439-1444.

[23] Minami $Y$, Nishida $N$, Kudo $M$. Therapeutic response assessment of RFA for HCC: contrast-enhanced US, CT and MRI. World J Gastroenterol 2014; 20:4160-4166. doi: 10.3748/wjg.v20.i15.4160.

[24] Imamura J, Tateishi R, Shiina S, Goto E, Sato T, Ohki T, et al. Neoplastic seeding after radiofrequency ablation for hepatocellular carcinoma. Am J Gastroenterol 2008;103:3057-3062. doi: 10.1111/j.1572-0241.2008. 02153.x

[25] Liu Z, Gao F, Yang G, Singh S, Lu M, Zhang T, et al. Combination of radiofrequency ablation with transarterial chemoembolization for hepatocellular carcinoma: an up-to-date meta-analysis. Tumour Biol 2014;35:7407-7413. doi: 10.1007/s13277-014-1976-z.

[26] Chen L, Sun J, Yang X. Radiofrequency ablation-combined multimodel therapies for hepatocellular carcinoma: Current status. Cancer Lett 2016; 370:78-84. doi: 10.1016/j.canlet.2015.09.020.

[27] Imamura $H$, Matsuyama $Y$, Miyagawa $Y$, Ishida $K$, Shimada R, Miyagawa S, et al. Prognostic significance of anatomical resection and des-gammacarboxy prothrombin in patients with hepatocellular carcinoma. $\mathrm{Br}$ J Surg 1999;86:1032-1038. doi: 10.1046/j.1365-2168.1999.01185.x.

[28] Ishii M, Mizuguchi T, Kawamoto M, Meguro M, Ota S, Nishidate T, et al. Propensity score analysis demonstrated the prognostic advantage of anatomical liver resection in hepatocellular carcinoma. World J Gastroenterol 2014;20:3335-3342. doi: 10.3748/wjg.v20.i12.3335.

[29] Nitta H, Beppu T, Imai K, Hayashi H, Chikamoto A, Baba H. Adjuvant hepatic arterial infusion chemotherapy after hepatic resection of hepatocellular carcinoma with macroscopic vascular invasion. World J Surg 2013;37: 1034-1042. doi: 10.1007/s00268-013-1957-1.

[30] Sun J], Wang K, Zhang CZ, Guo WX, Shi J, Cong WM, et al. Postoperative adjuvant transcatheter arterial chemoembolization after Ro hepatectomy improves outcomes of patients who have hepatocellular carcinoma with microvascular invasion. Ann Surg Oncol 2016;23:1344-1351. doi: 10.1245/ s10434-015-5008-z.

[31] Furtado R, Crawford M, Sandroussi C. Systematic review and meta-analysis of adjuvant $\mathrm{i}(131)$ lipiodol after excision of hepatocellular carcinoma. Ann Surg Oncol 2014;21:2700-2707. doi: 10.1245/s10434-014-3511-2. 